\title{
Towards Energy Saving Wireless Body Sensor Networks in Health Care Systems
}

\author{
B.Otal*, L.Alonso*, Ch. Verikoukis**, \\ * Department of Signal Theory and Communications, Technical University of Catalonia (UPC) \\ e-mail: bego otal@yahoo.es, luisg@tsc.upc.edu \\ ** Telecommunications Technological Center of Catalonia (CTTC) \\ e-mail: cveri@cttc.es
}

\begin{abstract}
The fact that the IEEE 802.15.4 MAC does not fully satisfy the strict wireless body sensor network (BSN) requirements in healthcare systems highlights the need for the design of new scalable MAC solutions, which guarantee low-power consumption to all specific sorts of body sensors and traffic loads. While taking the challenging healthcare requirements into account, this article aims at the study of energy consumption in BSN scenarios. For that purpose, the IEEE 802.15.4 MAC limitations are first examined and other potential MAC layer alternatives further explored. Our intent is to introduce energy-aware radio activation polices into a highperformance distributed queuing medium access control (DQ MAC) protocol and evaluate its energy-saving achievements, as a function of the network load. To do so, a fundamental energy-efficiency theoretical analysis for DQ MAC protocols is hereby for the first time provided. By means of computer simulations, its performance is validated using IEEE 802.15.4 MAC system parameters. The achieved outcome shows that the proposed DQ MAC scheme outperforms IEEE 802.15.4 MAC energy efficiency in all possible BSN scenarios.
\end{abstract}

Keywords - Body sensor networks, distributed queuing, energy efficiency, medium access control, power consumption

\section{INTRODUCTION}

Although the challenges faced by wireless body sensor networks (BSNs) in healthcare environments are in a certain way similar to those already existing in current wireless sensor networks (WSNs), there are intrinsic differences which require special attention [1]. For instance, human body monitoring may be achieved by attaching sensors to the body's surface as well as implanting them into tissues for a more accurate clinical practice. Hence, some of these newly emerged challenges due to healthcare requirements, range from low latency and high reliability, to low power consumption in order to protect human tissue. One of the major concerns in BSNs is that of extremely energy efficiency, which is also the key to extend the lifetime of battery-powered body sensors, reduce maintenance costs and avoid invasive procedures to replace battery in the case of implantable devices.

The MAC layer is responsible for coordinating channel accesses, by avoiding collisions and scheduling data transmissions, to maximize throughput efficiency at an acceptable packet delay and minimal energy consumption. While taking healthcare requirements into consideration, in this article, we concentrate on the evaluation of energy consumption, where using a number of wireless on-body sensors for regular patient monitoring, as for instance electrocardiography sensors (ECG) in hospital settings, is required. In this context, among all IEEE 802 standards available today, the IEEE 802.15.4 (802.15.4) [2] is regarded as the technology of choice for most BSN research studies [1],[3],[4]. However, even though the 802.15.4 MAC consumes very low power, the figures may not reach the levels required in BSNs [3],[4]. That is the reason why there exists the need to explore other MAC potential candidates for future BSNs.

The 802.15.4 MAC accepts three network topologies: star, peer-to-peer and cluster-tree. Our focus is here on 1-hop starbased BSNs, where a body area network (BAN) coordinator is elected. In a hospital BSN, the BAN coordinator can be a central care unit linked to a number of ward-patients wearing several body sensors (see Fig. 1). Communication from body sensors to BAN coordinator (uplink), from BAN coordinator to body sensors (downlink), or even from body sensor to body sensor (ad hoc) is possible. In the following, we study uplink and downlink communication, which occurs more often than ad hoc communication for regular patient monitoring BSNs.

In a 802.15.4 star-based network, the beacon mode appears to allow for the greatest energy efficiency. Indeed, it allows the transceiver to be completely switched off up to $15 / 16$ of the time when nothing is transmitted/received, while still allowing the transceiver to be synchronized to the network and able to transmit or receive a packet at any time [5]. The beacon mode introduces the so-called superframe structure. The inter-beacon period is partially or entirely occupied by the superframe, which is divided into 16 slots. Among them, there are at most 7 guaranteed time slots (GTS), (i.e. they are dedicated to specific nodes), which form the contention free period (CFP) [2]. This functionality targets very low latency applications, but it is not scalable in BSNs, since the number of dedicated slots is not sufficient [3]. In such conditions, the use of the contention access period (CAP) is required; where channel accesses in the uplink are coordinated by a slotted carrier sense multiple access mechanism with collision avoidance (CSMA/CA). Nevertheless, as we will see later, the CSMA/CA mechanism has a significant impact on the overall energy performance.

Next section follows with a brief overview of the most relevant distributed queuing MAC (DQ MAC) protocols. Section 3 introduces significant MAC protocol enhancements to minimize energy consumption in BSNs and the adopted energy-aware radio activation policies are presented in Section 4. Next, a fundamental energy-efficiency theoretical analysis for DQ MAC protocols is newly provided in Section 5. Simulation results are displayed in Section 6 to evaluate DQ MAC 
overall energy performance, as a function of 802.15.4 MAC system parameters within BSN scenarios. The last section concludes the paper.

\section{OVERVIEW OF Distributed QUEUING MAC PROTOCOLS}

This section highlights the basic features related to distributed queuing (DQ) MAC protocols that are essential for the understanding of the new energy-saving enhancements proposed in this article. The main characteristic of a DQ MAC protocol is that it behaves as a random access mechanism under low traffic conditions, and switches smoothly and automatically to a reservation scheme when the traffic load grows. That is, DQ MAC protocols show a near-optimum performance independent of the amount of active terminals and traffic load.

Let us consider a star-based topology with several nodes and a network coordinator, following DQRAP original description [8], the time axis is divided into an "access subslot" that is further divided into access minislots $(\mathrm{m})$, and a "data subslot". The basic idea is to concentrate user access requests in the access minislots, while the "data subslot" is devoted to collision-free data transmissions. The DQRAP analytical model approaches the delay and throughput performance of the theoretical optimum queuing systems $\mathrm{M} / \mathrm{M} / 1$ or $\mathrm{G} / \mathrm{D} / 1$, depending on the traffic distribution. Hence, DQ MAC protocols can be modeled as if every station in the system maintains two common logical distributed queues - the collision resolution queue (CRQ) and the data transmission queue (DTQ) -, physically implemented as four integers in each station; two station-dependant integers that represent the occupied position in each queue; and, two further integers shared among all stations in the system that visualize the total number of stations in each queue, CRQ and DTQ. The CRQ controls station accesses to the collision resolution server (the access minislots), while the DTQ is in charge of the data server (the "data subslot"). This provides a collision resolution tree algorithm that proves to be stable for every traffic load even over the system transmission capacity. Note that the number of access minislots is implementation dependant, but we are formally using 3 access minislots, following the original DQRAP structure and argumentation for maximizing its throughput performance [6]-[8]. A DQ MAC protocol consists of several strategic rules, independently performed by each station by managing the aforementioned four integers (i.e. corresponding to the two distributed queues, CRQ and DTQ) [8], which answer: i) 'who' transmits in the data slot and 'when', ii) 'who' sends an access request sequence in the minislots $(m)$ and 'when'; and iii) 'how' to actualize their positions in the queues.

Hence, the promising behavior of DQRAP in [6], [8], and similarly of DQCA in [7], in terms of delay and nearoptimum throughput achievements (i.e. allowing high reliability), evokes the idea to further explore DQ MAC protocols in terms of energy consumption under BSN healthcare scenarios. This favorable behavior is especially achieved thanks to the inherent protocol performance at eliminating collisions in data transmissions and minimizing the overhead of contention procedures (i.e. carrier sensing and back-off periods) with respect to CSMA/CA. Based on that, we introduce energyefficient enhancements to allow radio activation policies and power management solutions for the proper use of DQ MAC in BSNs. Additionally, we propose here a new basic energyefficiency theoretical analysis for DQ MAC protocols, and by means of computer simulations, we evaluate its performance using 802.15.4 MAC system parameters.

\section{DQ MAC ENERGY-SAVING ENHANCEMENTS FOR BSNS}

Fig. 1 shows the superframe format of a DQ MAC protocol proposal for star-based BSNs with several ward-patients wearing a number of body sensors that communicate to a central care unit (i.e. BAN coordinator). The complete energy-saving superframe structure comprises two differential parts; (i) from body sensors to BAN coordinator (uplink), with a CAP and a CFP. The CAP is further divided into $\mathrm{m}$ access minislots, whereas the CFP is devoted to collision-free data packet transmissions, and, (ii) from BAN coordinator to body sensors (downlink) using the feedback frame, which contains several strategic fields.

In fact, the DQ MAC superframe is bounded by the feedback packet (FBP) contained in the Fig. 1 portrayed feedback frame, which is broadcasted by the BAN coordinator. Similar to the 802.15.4 MAC superframe format, one of the main uses of the FBP is to synchronize the attached body sensors to the BAN coordinator. The FBP always contains relevant MAC control information (i.e. corresponding also to the protocol rules), which is essential for the right functioning of all body sensors in the BSN. When a body sensor wishes to transfer data, it first waits for the FBP. After synchronization, it independently actualizes the integer counters, by applying a set of rules that determine its position in the protocol distributed queues, CRQ and DTQ. At the appropriate time, the body sensor transmits either an access request sequence (ARS) in one of the randomly selected access minislots (within the CAP), or its data packet in the "data slot" (within the CFP). The BAN coordinator may acknowledge the successful reception of the data packet by sending an optional acknowledgment frame (ACK). This sequence is summarized in Fig. 1.

The main differences of this energy-saving DQ MAC superframe format with respect to previous DQ MAC ones are the following:

- A new preamble (PRE) between the ACK and the FBP is introduced to enable synchronization after power-sleep modus (i.e. idle or shutdown). That is to say that the body sensors, which are not supposed to be ACK recipients, are longer maintained in power-sleep modus, as later detailed.

- Further, the FBP is here of fixed length (i.e. independently of the number of body sensors in the BSN) and contains two strategic fields for specific energy-aware radio activation policies and power management solutions. These are the modulation and coding scheme (MCS) and the length of the 
data packet to be transmitted in the next CFP. This facilitates scalable power management processes for future multi-rate medical applications, and allows the use of a flexible CFP (i.e. data packets of different lengths for applicationoriented medical body sensors).

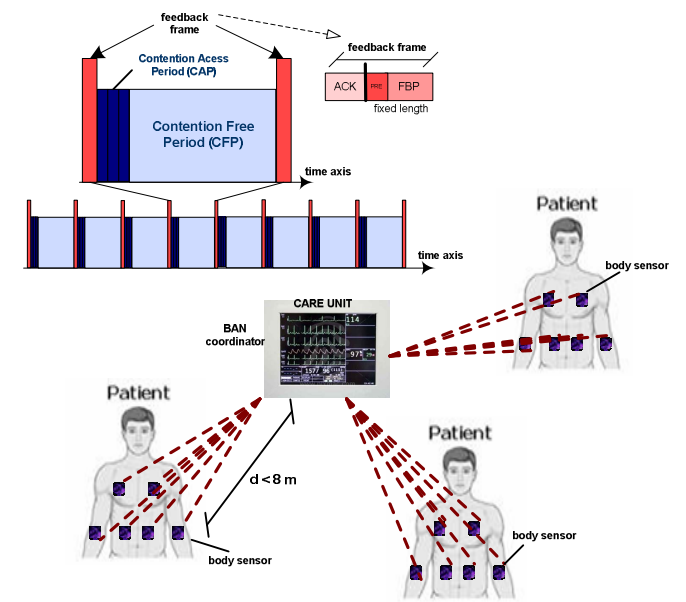

Figure 1. A star-based BSN with DQ MAC superframe format

\section{DQ MAC RADIO ACTIVATION POLICIES}

To be able to asses the average energy consumption of a body sensor in a BSN, we must first characterize the instantaneous power consumption of the transceiver, when operating in different states. Apart from the transmit and receive modes, a transceiver supports two further states: shutdown, when the clock is switched off and the chip is completely deactivated waiting for a start-up strobe; and, idle, when the clock is turned on and the chip can receive commands, for example, to turn on the radio circuitry [5].

Fig. 2 illustrates our enhanced DQ MAC superframe format to allow different power management scenarios to body sensors using an energy-aware radio activation policy under BSNs. Note that each time slot is characterized by a different power consumption modus (i.e. transmit, receive, idle, and shutdown). As previously mentioned, each body sensor synchronizes to the BSN thanks to the novel preamble sequence (PRE) of duration $t_{P R E}$ after a period in idle mode. Thereafter, it receives the required system information via the FBP of duration $t_{F B P}$ for updating the distributed queues, CRQ and DTQ [6]-[8]. After each FBP, a short inter-frame space $t_{I F S}$ is left to allow the MAC layer to process the data received from the PHY layer, like in 802.15.4 [2].

Active body sensors involved in the access procedure like in scenarios (1) and (2) start by sending a ARS, here of duration length $t_{A R S}$, in one of the randomly selected access $m i$ nislots [8]. Prior to that, these body sensors should have switched its radio from idle to transmit mode, which take them a transition time $t_{i a}$ for body sensor radio wake-up (i.e. from idle to active modes [5]).

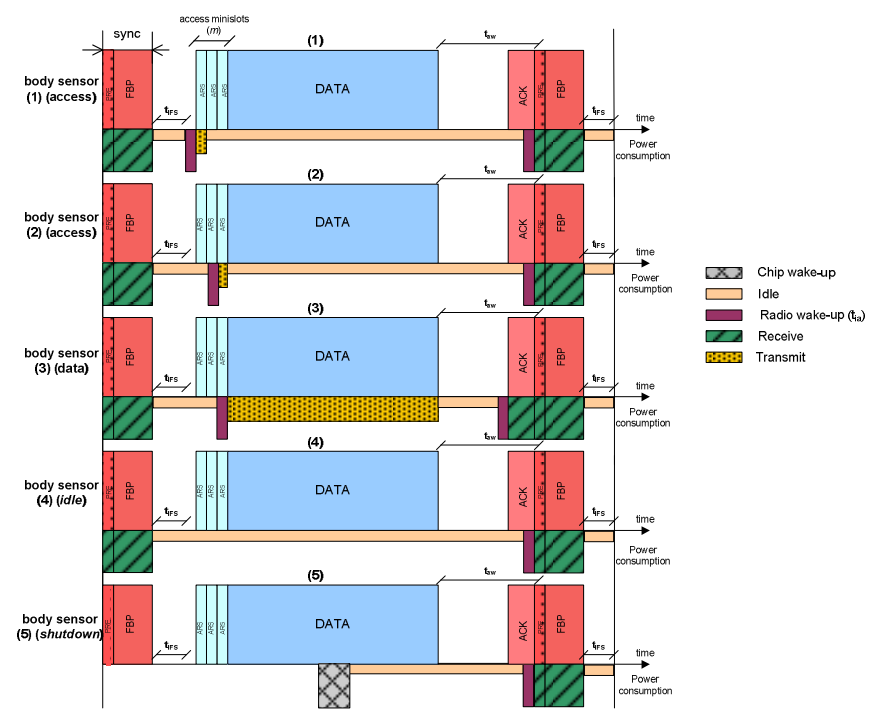

Figure 2. Power management scenarios in BSNs

Next, scenario (3) depicts the transmission of a previously granted packet of average duration length $\bar{t}_{D A T A}$ preceded by the transition time $t_{i a}$. If the packet is received correctly, an acknowledgement (ACK) of duration $t_{A C K}$ is sent back to the transmitting body sensor followed by the FBP (and PRE) after a maximum time $t_{a w}-t_{A C K}$, during which the receiver turns its radio to idle mode to save energy. In [2], $t_{a w}$ is characterized as the maximum time to wait for an ACK. Scenario (4) shows how an active body sensor waiting in idle mode synchronizes through the PRE to receive the FBP. Finally, scenario (5) portrays how a body sensor in shutdown state wakes up and waits for some time in idle mode to synchronize through the PRE and get the FBP to update the state of the CRQ and DTQ queues [6]-[8].

\section{DQ MAC ENERGY-EFFICIENCY ANALYSIS}

Let us now define $P_{t x}, P_{r x}$ and $P_{i d l e}$ as the power consumption in transmit, receive and idle modes respectively, and similarly, $\bar{T}_{t x}, \bar{T}_{r x}$ and $\bar{T}_{i d l e}$, as the average time a body sensor spends in each of the aforementioned modes within the queuing system (i.e. CRQ and DTQ). Thus, the average consumed energy per information bit for every active body sensor in the BSN can be expressed as $\bar{E}_{b i t}=\bar{E}_{F R A M E} / L_{b i t}$, where $L_{b i t}$ corresponds to the payload data length in bits, and

$$
\bar{E}_{\text {FRAME }}=P_{t x} \cdot \bar{T}_{t x}+P_{r x} \cdot \bar{T}_{r x}+P_{i d l e} \cdot \bar{T}_{i d l e} .
$$

The average time in transmit, receive and idle mode can be computed as, 


$$
\begin{aligned}
& \bar{T}_{t x}=\bar{n}_{t x} \cdot\left(t_{A R S}+t_{i a}\right)+\bar{t}_{D A T A}+t_{i a}, \\
& \bar{T}_{r x}=\bar{n}_{\text {waiting }} \cdot\left(t_{P R E}+t_{F B P}+t_{i a}\right)+t_{A C K}, \\
& \bar{T}_{\text {idle }}=\bar{n}_{\text {waiting }} \cdot\left[\bar{T}_{F R A M E}-\left(t_{P R E}+t_{F B P}\right)\right] .
\end{aligned}
$$

The average duration of the DQ MAC time superframe, $\bar{T}_{F R A M E}$, derived from Fig. 2 is characterized as,

$$
\bar{T}_{F R A M E}=m \cdot t_{A R S}+\bar{t}_{D A T A}+t_{a w}+t_{P R E}+t_{F B P}+t_{I F S},
$$

where $m$ corresponds to the number of minislots used in the current DQ MAC superframe structure, and $t_{A R S}, \bar{t}_{D A T A}$, $t_{a w}, t_{A C K}, t_{P R E}, t_{F B P}, t_{I F S}$ and $t_{i a}$ have been previously defined following the illustration example of power management scenarios in Fig. 2. Here, we specify $\bar{n}_{\text {waiting }}$ and $\bar{n}_{t x}$, as the total average number of slot time frames waiting in the whole queuing system (i.e. CRQ and DTQ), and, the average number of slot time frames used to transmit an ARS in the CRQ system, respectively. Their concrete characterization is not straightforward, but both numbers can be derived from DQRAP original delay theoretical analysis in [9]. The following performance evaluation compares DQ MAC energyefficient analysis with computer simulations.

\section{PERformance EVALUATION}

The performance of the previously studied energyefficiency analysis is validated via MATLAB computer simulations, by implementing the DQ MAC protocol [6]-[8], within a star-based BSN scenario, as the relative traffic load increases until saturation conditions. Relative traffic load is here defined, as the ratio of generated data packets per iteration. The traffic load (portrayed in the $\mathrm{x}$-axis) rises by increasing the number of active body sensors in the BSN in each simulation. Note that all body sensors follow a Poisson traffic distribution, since we consider here a generalized case scenario. The energy consumption (shown in the y-axis) is computed considering every body sensor spent time and power consumption in each of the aforementioned states (i.e. transmit, receive and idle) following DQ MAC procedure in our simulated BSN scenario. Thus, the energy consumption per information bit is defined as the ratio of the total energy consumption per body sensor and per payload packet length (i.e. information bit).

\subsection{Reference Scenario}

Every active body sensor is supposedly located at a random distance $d$ from the BAN coordinator, as portrayed in Fig.1. The channel link implementation is based on the path loss model of the 802.15.4 standard [2], where the average received power is expressed as a function of an arbitrary T-R separation distance $d<8$ meters (i.e. within a hospital set- ting). In our simulations, the time-variant received signal also includes Additive White Gaussian Noise (AWGN) and the effect of log-normal shadowing, assuming the channel is coherent within the transmission of a DQ MAC superframe, like in indoor environments. The reference BSN scenario is characterized by the system parameters corresponding to the standardized 802.15.4 MAC default values in the upper frequency band $2.4 \mathrm{GHz}$ at the fixed data rate $250 \mathrm{~Kb} / \mathrm{s}$ [2]. Following the illustration of DQ MAC superframe structure in Fig. 2, we choose the longest data payload lengths $(L)$ of 80,100 and 120 bytes, to minimize the PHY (6 bytes) and MAC ( 8 bytes) headers overhead per information bit. Further, a packet may be corrupted by bit errors due to noise. Hence, a body sensor waits for an ACK (11 bytes) for a maximum time of $t_{a w}-t_{A C K}$, where $t_{a w}$ is limited to $864 \mu \mathrm{s}$, as defined in [2]. The synchronization PRE corresponds to 4 bytes and it is followed by the FBP of 11 bytes, similar to a beacon [2]. Additionally, we use 3 access minislots, like in [6]-[8], and an ARS occupies hereby the same size as a preamble sequence (i.e. 4 bytes), which is a worst case assumption. All these figures are summarized in Table 1.

Table 1. IEEE 802.15.4 and DQ MAC Parameter Values

\begin{tabular}{|c|c||c|c|}
\hline Parameter & Value & Parameter & Value \\
\hline PHY header & 6 bytes & ACK & 11 bytes \\
\hline MAC header & 9 bytes & Beacon & 11 bytes \\
\hline Data payload & 80 to 120 bytes & $t_{a w}$ & $864 \mu \mathrm{s}$ \\
\hline Data rate & $250 \mathrm{~Kb} / \mathrm{s}$ & $t_{I F S}$ & $192 \mu \mathrm{s}$ \\
\hline \multicolumn{4}{|c||}{ DQ MAC } \\
\hline Preamble & 4 bytes & $m$ & 3 \\
\hline FBP & 11 bytes & $t_{A R S}$ & $100 \mu \mathrm{s}$ \\
\hline
\end{tabular}

In order to make a fair comparison, all used transceiver power consumption values are formalized as in [5] and portrayed in Table 2 . Note that the power consumption in transmission mode is this time for a transmit power of $-5 \mathrm{dBm}$. That is the value used in [5] analytical results, which we use for our comparison with DQ MAC energy-efficiency analysis.

Table 2. Transceiver Power Consumption

\begin{tabular}{|c|c|c|}
\hline$P_{t x}$ & $P_{r x}$ & $P_{\text {idle }}$ \\
\hline $22.09 \mathrm{~mW}$ & $35.23 \mathrm{~mW}$ & $712 \mu \mathrm{W}$ \\
\hline
\end{tabular}

\subsection{Analytical and Simulation Results}

The analytical and simulation results are portrayed hereafter. Fig. 3 portrays the analytical results of the energy consumption per information bit of DQ MAC versus the theoretical analysis of 802.15.4 MAC in [5], as the relative traffic load in the BSN increases. It can be seen, that the use of DQ MAC outperforms 802.15 .4 MAC by reducing a $36.65 \%$ the energy consumption per information bit, when the relative traffic load is as high as $80 \%$. 


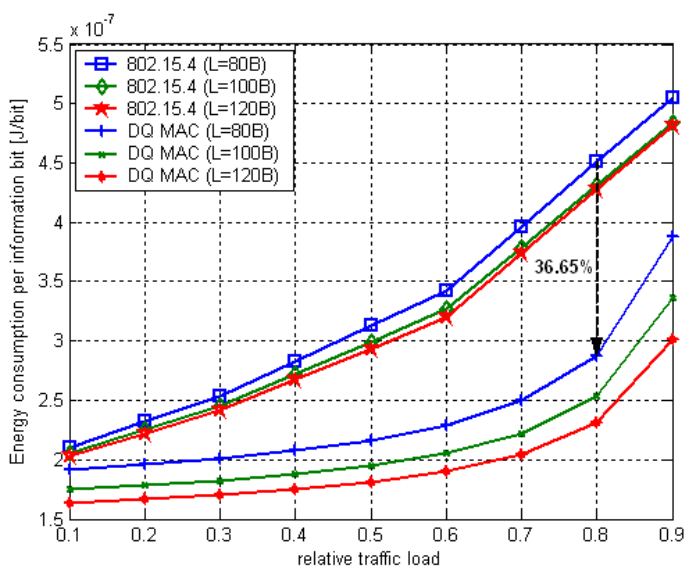

Figure 3. $D Q M A C$ vs. 802.15.4 MAC energy consumption per information bit - Analytical results

Eventually, analytical and simulated results of DQ MAC energy consumption per information bit are depicted in Fig. 4. Here, it can be seen the excellent protocol performance even for the highest traffic load between $80 \%$ and $90 \%$, which remains under $350 \mathrm{~nJ} / \mathrm{bit}$. Thus, the simulation results corroborate the accuracy of the newly introduced theoretical analysis in terms of energy efficiency. They also show the appropriate scalability of DQ MAC energy-saving performance for future BSN scenarios, while fulfilling healthcare stringent power consumption requirements.

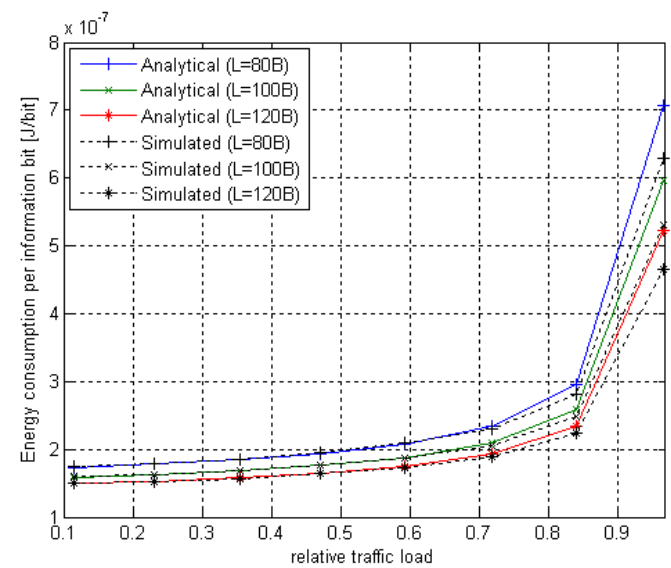

Figure 4. $D Q M A C$ energy consumption per information bit - Analytical vs. Simulated

\section{CONCLUSIONS}

In this article, we have been evaluating IEEE 802.15.4 MAC limitations under new challenging healthcare requirements for wireless body sensor networks (BSNs). Further, a new energy-efficiency theoretical analysis for an enhanced distributed queuing medium access control protocol has been introduced, as a potential candidate for future BSNs. For that purpose, energy-aware radio activation policies are first introduced in order to allow power management regulation to minimize the energy consumption per information bit. The analytical study has been validated by simulation results, which have shown that the proposed mechanism outperforms IEEE 802.15.4 MAC energy-efficiency for all traffic loads in a generalized BSN scenario. This favorable energy-efficient behavior is especially achieved thanks to the inherent protocol performance at eliminating collisions in data transmissions, while minimizing the control overhead and hence the overall energy consumption per information bit.

\section{ACKNOWLEDGMENT}

This work was partially funded by the Research Projects PERSEO (TEC2006-10459/TCM), 2009 SGR 940, CENTENO (TEC2008-06817-C0202 ) and COOLNESS (218163-FP7-PEOPLE).

\section{REFERENCES}

[1] G-Z. Yang (Ed.), Body Sensor Networks, Springer-Verlag London Limited 2006.

[2] IEEE Std. 802.15.4-2003, "IEEE Standards for Information Technology Part 15.4: Wireless Medium Access Control (MAC) and Physical Layer (PHY) Specifications for Low-Rate Wireless Personal Area Networks (LR-WPANs)", 1st October 2003.

[3] B. Zhen, H-B. Li, and R. Kohno, "IEEE Body Area Networks for Medical Applications", in Proc. IEEE $4^{\text {th }}$ International Symposium on Wireless Communication Systems (ISWCS 2007), Trondheim, Norway, 17-19 Oct 2007, pp. 327-331.

[4] P. Kumar, M. Günes, A.B. Almamou, and J. Schiller, "Realtime, Bandwidth, and Energy Efficient IEEE 802.15.4 for Medical Applications", in Proc. 7th GI/ITG KuVS Fachgespräch "Drahtlose Sensornetze", FU Berlin, Germany, September 2008.

[5] B. Bourgard, F. Catthoor, D.C. Daly, A. Chandrakasam and W. Dehaene, "Energy Efficiency of the IEEE 802.15.4 Standard in Dense Wireless Microsensor Networks: Modeling and Improvement Perspectives", in Proc. IEEE Design Automation and Test in Europe Conference and Exhibition, Calgary, Canada, March 2005, pp. 196-201.

[6] H.J. Lin and G. Campbell, "Using DQRAP (Distributed Queuing Random Access Protocol) for local wireless communications", in Proc. Wireless '93, Calgary, Canada, July 1993, pp. 625-635.

[7] L. Alonso, R. Ferrús, and R. Agustí, "WLAN Throughput Improvement via Distributed Queuing MAC," IEEE Communication Letters, vol. 9, no. 4, Apr. 2005, pp. 310-12.

[8] X. Xu, G. Campbell, "A Near Perfect Stable Random Access Protocol for a Broadcast Channel", in Proc. IEEE Communications, Discovering a New World of Communications (SUPERCOMM/ICC'92), Chicago, USA, June 1992, vol. 1, pp. $0370-$ 0374.

[9] Zhang and G. Campbell, "Performance Analysis of Distributed Queuing Random Access Protocol - DQRAP", August 1993, DQRAP Research Group. 